\title{
Autoimmunity and Benefit from Trastuzumab Treatment in Breast Cancer: Results from the HERA Trial
}

\author{
AMIR SONNENBLICK ${ }^{1}$, ANDREW BAILEY $^{2}$, BEATRICE UZIELY $^{3}$, MICHAEL UNTCH $^{4}$, IAN SMITH $^{5}$, \\ LUCA GIANNI $^{6}$, JOSE BASELGA ${ }^{7}$, CHRISTIAN JACKISCH $^{8}$, DAVID CAMERON $^{9}$, RICHARD BELL $^{10}$, \\ DIMITRIOS ZARDAVAS ${ }^{11}$, NEDAL AL-SAKAFF ${ }^{12}$, RICHARD D. GELBER ${ }^{13}$, MITCH DOWSETT ${ }^{14}$, \\ BRIAN LEYLAND-JONES ${ }^{15}$, MARTINE J. PICCART-GEBHART ${ }^{16}$ and EVANDRO DE AZAMBUJA ${ }^{16}$ \\ ${ }^{1}$ Institute of Oncology, Tel Aviv Sourasky Medical Center, Tel Aviv, Israel; \\ Sackler Faculty of Medicine, Tel Aviv University, Tel Aviv, Israel; \\ ${ }^{2}$ Frontier Science, Kingussie, U.K.; \\ ${ }^{3}$ Hadassah-Hebrew University Medical Center, Jerusalem, Israel; \\ ${ }^{4}$ Klinik für Gynäkologie und Geburtshilfe, Interdisziplinäres Brustzentrum, \\ HELIOS Klinikum Berlin Buch, Berlin, Germany; \\ ${ }^{5}$ Breast Unit, The Royal Marsden Hospital NHS Foundation Trust, London, U.K.; \\ ${ }^{6}$ Department of Medical Oncology, San Raffaele Hospital, Scientific Institute, Milan, Italy; \\ ${ }^{7}$ Memorial Sloan-Kettering Cancer Center, New York, NY, U.S.A.; \\ ${ }^{8}$ Department of Gynecology and Obstetrics, Sana Klinikum Offenbach, Offenbach, Germany; \\ ${ }^{9}$ Western General Hospital, University of Edinburgh Cancer Research Centre, Edinburgh, U.K.; \\ ${ }^{10}$ Deakin University, Waurn Ponds, VIC, Australia; \\ ${ }^{11}$ Breast International Group (BIG), Brussels, Belgium; \\ ${ }^{12}$ F. Hoffmann-La Roche, Basel, Switzerland; \\ ${ }^{13}$ Department of Biostatistics and Computational Biology, Dana-Farber Cancer Institute, \\ Harvard TH Chan School of Public Health and Frontier Science and Technology Research Foundation, \\ Harvard Medical School, Boston, MA, U.S.A.; \\ ${ }^{14}$ Academic Department of Biochemistry, Royal Marsden Hospital, London, U.K.; \\ ${ }^{15}$ Department of Molecular and Experimental Medicine, Avera Cancer Institute, Sioux Falls, SD, U.S.A.; \\ ${ }^{16}$ Institut Jules Bordet, Université Libre de Bruxelles, Brussels, Belgium
}

\begin{abstract}
Background/Aim: This study sought to determine whether an autoimmune background could identify patients with HER2-positive early breast cancer $(E B C)$ who derive differential benefit from primary adjuvant trastuzumab-based therapy. Patients and Methods: HERA is an international randomized trial of 5,102 women with HER2-positive EBC, who were enrolled to either receive adjuvant trastuzumab or not. In this exploratory analysis, the interaction between autoimmune history and the magnitude of trastuzumab benefit was evaluated. Results: A total of 5,099 patients were included
\end{abstract}

This article is freely accessible online.

Correspondence to: Dr. Evandro de Azambuja, Institut Jules Bordet, Bld de Waterloo, 121(7th Floor), 1000 Brussels, Belgium. Tel: +32 025417244, Fax: +32 025413477, e-mail: evandro.azambuja@bordet.be

Key Words: Autoimmunity, trastuzumab, HER2, breast cancer. in the current analysis. Among them, 325 patients (6.4\%) had autoimmune disease history, 295 of whom had active disease. Patients were randomly assigned to trastuzumab or notrastuzumab groups. Similar reductions in the risk of events in patients with and without autoimmune history were observed (interaction $p=0.95$ for disease-free survival, and $p=0.62$ for overall survival). Conclusion: No evidence of a differential benefit from trastuzumab in patients with a medical history of autoimmune disease was found.

The ErbB2/HER2 (human epidermal growth factor receptor 2) oncogene encoding for a member of the epidermal growth factor family, was found to be amplified in about $20 \%$ of breast cancer patients and is associated with a shorter time to relapse and a lower survival rate $(1,2)$. Trastuzumab, a monoclonal antibody against HER2 received approval for the treatment of patients with HER2-positive metastatic breast cancer on the basis of a pivotal trial (3). At the turn of the $21 \mathrm{st}$ century, a number of randomized prospective clinical trials 
were launched to evaluate the role of trastuzumab given in addition to chemotherapy in the adjuvant setting. These studies generated highly consistent results, showing that the addition of trastuzumab to chemotherapy decreased recurrence by approximately $50 \%$ and mortality by $30 \%$ (4-7).

One of these trials was the HERA (BIG 1-01) trial, which randomized 5,102 patients who were enrolled after completion of their postoperative chemotherapy. The final analysis of an 11 years follow-up showed that the addition of 1 year of adjuvant trastuzumab resulted in a constant $24 \%$ relative reduction in the risk of a disease-free survival event (DFS), and a $26 \%$ relative reduction in the risk of death (5).

In the last few decades the role of the immune system in the outcome of cancer treatment has moved rapidly from preclinical evidence to clinical practice. The innate immune system involves activation of dendritic cells, natural killer cells, granulocytes as well as other cells and the complement system, and is associated with a rapid non-specific quick immune response without the need of memory or previous exposure (8). On the other hand, the adaptive immune response develops slowly and is mediated by a response to a specific antigen by $\mathrm{B}$ cells and $\mathrm{T}$ cells. Adaptive and innate immune mechanisms are essential players in the therapeutic effects of monoclonal antibody-based antiHER2 therapy (9-11). Higher levels of tumor infiltrating lymphocytes (TILs) in the primary tumor at diagnosis are associated with increased benefit from trastuzumab (12).

Autoimmune diseases may cause significant and chronic morbidity, and women have a 2.7-times greater risk than men to develop an autoimmune disease (13). In this HERA exploratory analysis, we sought to examine whether autoimmunity background is associated with a different outcome in patients treated with primary trastuzumab-based therapy.

\section{Patients and Methods}

Study patients. HERA is an open-label, phase III, international multicenter randomized trial. A detailed description of the trial, its regimens, and key eligibility criteria are provided in the original report (4). Briefly, between Dec 7, 2001, and June 20, 2005, 5,102 patients were recruited and randomly assigned $(1: 1: 1)$ to one of the three treatments arms: observation (without trastuzumab), adjuvant treatment of trastuzumab for 1 year or for 2 years.

The comparison of the trastuzumab arms versus observation was based on the intention-to-treat (ITT) principle, after exclusion of three patients (one from each group) because of no record of written informed consent (Figure 1). To be eligible, participants had to have central laboratory (Kassel, Germany) confirmation of locally assessed HER2-positive disease and left ventricular ejection fraction (LVEF) of at least 55\% after completion of chemotherapy and radiotherapy if applicable. History of autoimmune diseases was prospectively collected in the clinical database and was retrieved for the sake of this analysis. Of note, this analysis was not preplanned as a part of the data analysis plan of the trial. The HERA trial was approved by the ethics committees of all participating sites and this exploratory analysis was approved by the HERA executive committee.

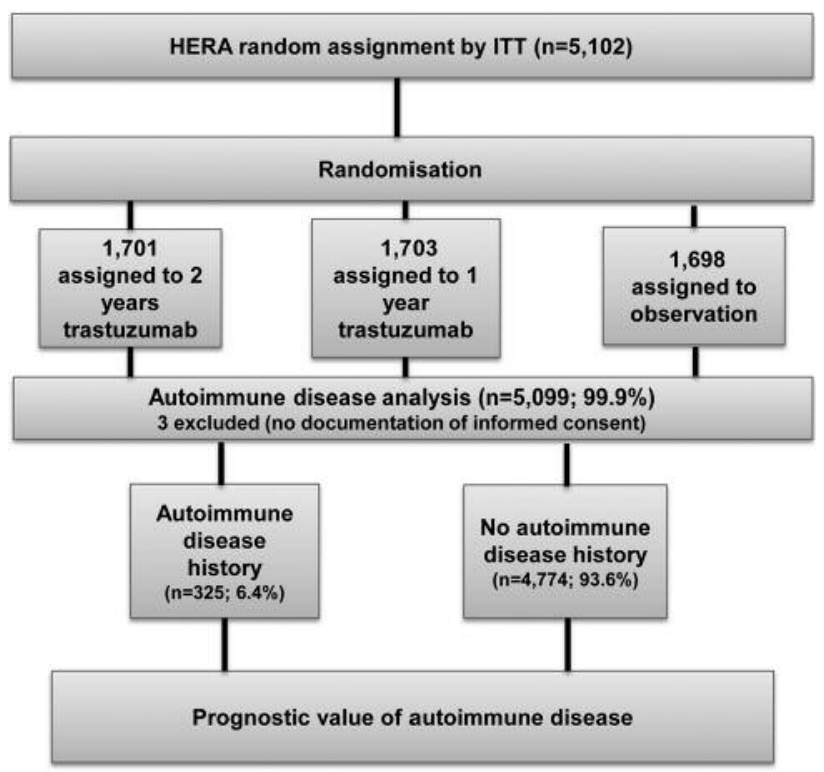

Figure 1. CONSORT diagram. Study flowchart shows the process of patient selection.

Statistical analysis. In the current analysis, we investigated the potential impact of autoimmune diseases on patient's outcome. We hypothesized that autoimmune history of the patients may elicit robust antitumor immune response, boosting the antitumor activity of trastuzumab, thus prolonging survival. Therefore, the a priori hypothesis was that patients with autoimmune disease background would have superior long-term outcomes when treated with trastuzumab. We evaluated the association between autoimmune background and classic clinicopathological factors using Chisquared tests. Autoimmune diseases as well as other comorbidities in the HERA database were coded using the WHO-DDE (World Health Organization Drug Dictionary Enhanced). This analysis was restricted to autoimmune predefined diseases for which there was direct or indirect evidence for autoimmune pathogenesis (13). Two survival endpoints were investigated: disease-free survival (DFS) and overall survival (OS), which were defined in the HERA study (4). In these exploratory analyses, the two trastuzumab treatment arms were combined. A Cox proportional hazards regression model was used to assess the interaction between autoimmune history and treatment $(1+2$ years trastuzumab and observation). Cox modelling was also used with the overall population and for the two autoimmune status sub-populations to estimate unadjusted hazard ratios (HR) and 95\% CIs, comparing $1+2$ years trastuzumab and observation.

\section{Results}

Association between autoimmune disease history and clinicopathological characteristics. The HERA clinical database was screened for preferred terms of predefined autoimmune comorbidities (13), for which there was clear direct or indirect evidence for autoimmune pathogenesis. All 
Table I. Summary of autoimmune disease at baseline.

\begin{tabular}{|c|c|c|c|c|}
\hline \multirow[b]{2}{*}{ Thyroid background } & \multirow[b]{2}{*}{ Autoimmune disease preferred term } & \multicolumn{2}{|c|}{ Diagnosis } & \multirow[b]{2}{*}{ Total $(\mathrm{N}=325)$} \\
\hline & & Active $(\mathrm{N}=295)$ & Not active $(\mathrm{N}=30)$ & \\
\hline \multirow[t]{2}{*}{ Hyperthyroidism } & Hyperthyroidism & 41 & 11 & 52 \\
\hline & Total & 41 & 11 & 52 \\
\hline \multirow[t]{4}{*}{ Hypothyroidism } & Hypothyroidic Goitre & 5 & 1 & 6 \\
\hline & Hypothyroidism & 213 & 5 & 218 \\
\hline & Primary Hypothyroidism & 1 & 0 & 1 \\
\hline & Total & 219 & 6 & 225 \\
\hline \multirow[t]{4}{*}{ Thyroiditis } & Autoimmune Thyroiditis & 7 & 1 & 8 \\
\hline & Thyroiditis & 2 & 3 & 5 \\
\hline & Thyroiditis Chronic & 1 & 0 & 1 \\
\hline & Total & 10 & 4 & 14 \\
\hline \multirow[t]{9}{*}{ No Thyroid Background } & Cutaneous Lupus Erythematosus & 2 & 0 & 2 \\
\hline & Lupus Nephritis & 1 & 0 & 1 \\
\hline & Multiple Sclerosis & 2 & 1 & 3 \\
\hline & Myasthenia Gravis & 1 & 1 & 2 \\
\hline & Rheumatoid Arthritis & 12 & 6 & 18 \\
\hline & Systemic Lupus Erythematosus & 2 & 1 & 3 \\
\hline & Type 1 Diabetes Mellitus & 4 & 0 & 4 \\
\hline & Vitiligo & 1 & 0 & 1 \\
\hline & Total & 25 & 9 & 34 \\
\hline
\end{tabular}

patients who were enrolled in the HERA trial were included in the current analysis $(\mathrm{N}=5,099)$, of whom 4,774 $(93.6 \%)$ patients had no history of autoimmune disease at baseline, while 325 patients $(6.4 \%)$ had autoimmune disease history (Figure 1).

A summary of the autoimmune diseases (preferred terms) that were detected in the HERA database are reported in Table I. Most of the autoimmune diseases were reported as active at baseline $(\mathrm{N}=295,90.7 \%)$ and the majority of them were associated with hypothyroidism ( $\mathrm{N}=219,67.4 \%)$. Table II lists the patients' characteristics according to autoimmune diseases history. There was some evidence that patients with an autoimmune background were more likely to be older $(p<0.001)$ and postmenopausal $(p<0.001)$ (Table II).

Prognostic value of the autoimmune background in trastuzumab treated patients. The association between autoimmune background and DFS and OS at a median follow-up of 11 years (IQR=10.09-11.53) was evaluated. During this follow-up period, 1,631 patients experienced a DFS event and 1,037 patients an OS event. One or two years of trastuzumab treatment of patients with no autoimmune history, compared to no trastuzumab, reduced the risk of DFS event (Hazard Ratio $(\mathrm{HR})=0.77,95 \%$ Confidence Interval $(\mathrm{CI})=0.69-0.85)$ and death $(0.74$, $95 \% \mathrm{CI}=0.65-0.84)$. In patients with autoimmune history treated with trastuzumab, the estimated magnitude of reduction in the risk of DFS or OS events was similar to that seen in the no-immune history group (DFS $\mathrm{HR}=0.76$, $95 \% \mathrm{CI}=0.51-1.12$; OS HR=0.65, 95\%CI $=0.40-1.07)$ (Table III). Evaluation of the interaction between autoimmune disease and treatment suggests that there is no evidence to reject the null hypothesis that there is no interaction (DFS $p=0.95$, OS $p=0.62$ ).

\section{Discussion}

In the present study, we sought to determine whether an autoimmune disease background could identify patients who derive different benefit from primary trastuzumab-based therapy. While there is a growing body of evidence that shows that the immune system contributes substantially to the therapeutic effects of trastuzumab, we were not able to demonstrate an interaction between autoimmune disease background and the level of benefit from trastuzumab treatment.

In HER2 positive breast cancer, the presence of TILs has been shown to have a prognostic value for improved probability of cure in early stages (14) and predictive for trastuzumab benefit (12). These TILs may represent a basic host anti-tumour immune response, which can be further boosted by immunotherapeutic agents. The presence of an autoimmune response is the defining characteristic of an autoimmune disease. Autoimmune diseases can be initiated by different antigens and despite their diverse etiology they are characterized by the presence of self-reactive $T$ lymphocytes (15) and the loss of inhibitory pathways in the 
Table II. Summary of patient's demographic and breast cancer characteristics according to autoimmune background.

\begin{tabular}{|c|c|c|c|}
\hline Characteristic & $\begin{array}{l}\text { Autoimmune history } \\
\qquad(\mathrm{N}=325)\end{array}$ & $\begin{array}{c}\text { No autoimmune history } \\
(\mathrm{N}=4774)\end{array}$ & $\begin{array}{l}p \text {-Value } \\
\text { chi-square }\end{array}$ \\
\hline \multicolumn{4}{|l|}{ Age at study entry (years) - no.(\%) } \\
\hline$<35$ & $11(3.4)$ & $367(7.7)$ & \multirow[t]{4}{*}{$<0.001$} \\
\hline $35-49$ & $111(34.2)$ & $2153(45.1)$ & \\
\hline $50-59$ & $130(40.0)$ & $1509(31.6)$ & \\
\hline$\geq 60$ & $73(22.5)$ & $745(15.6)$ & \\
\hline \multicolumn{4}{|l|}{ Previous (neo)adjuvant chemotherapy - no.(\%) } \\
\hline No anthracycline & $13(4.0)$ & $289(6.1)$ & \multirow[t]{3}{*}{0.168} \\
\hline Anthracycline but no taxane & $217(66.8)$ & $3252(68.1)$ & \\
\hline Anthracycline and taxane & $95(29.2)$ & $1233(25.8)$ & \\
\hline \multicolumn{4}{|l|}{ Menopausal status - no.(\%) } \\
\hline Premenopausal & $38(11.7)$ & $679(14.2)$ & \multirow[t]{3}{*}{$<0.001$} \\
\hline Uncertain & $102(31.4)$ & $1961(41.1)$ & \\
\hline Postmenopausal & $185(56.9)$ & $2134(44.7)$ & \\
\hline \multicolumn{4}{|l|}{ Pathological tumor size $(\mathrm{cm})-$ no. $(\%)$} \\
\hline $0-2$ & $130(40.0)$ & $1873(39.2)$ & \multirow[t]{5}{*}{0.907} \\
\hline $2-5$ & $138(42.5)$ & $2087(43.7)$ & \\
\hline$>5$ & $16(4.9)$ & $257(5.4)$ & \\
\hline Not assessed (neoadjuvant chemotherapy) & $38(11.7)$ & $525(11.0)$ & \\
\hline Missing size & $3(0.9)$ & $32(0.7)$ & \\
\hline \multicolumn{4}{|l|}{ Pathological nodal status - no. $(\%)$} \\
\hline Negative & $103(31.7)$ & $1543(32.3)$ & \multirow[t]{5}{*}{0.966} \\
\hline 1-3 Positive nodes & $95(29.2)$ & $1369(28.7)$ & \\
\hline$\geq 4$ Positive nodes & $89(27.4)$ & $1336(28.0)$ & \\
\hline Not assessed (neoadjuvant chemotherapy) & $38(11.7)$ & $525(11.0)$ & \\
\hline Missing & $0(0.0)$ & $1(0.0)$ & \\
\hline \multicolumn{4}{|l|}{ Local hormone-receptor status - no.(\%) } \\
\hline Negative & $174(53.5)$ & $2354(49.3)$ & \multirow[t]{2}{*}{0.152} \\
\hline Positive & $151(46.5)$ & $2420(50.7)$ & \\
\hline \multicolumn{4}{|l|}{ Histological grade of tumor - no.(\%) } \\
\hline G1 & $3(0.9)$ & $103(2.2)$ & \multirow[t]{5}{*}{0.084} \\
\hline $\mathrm{G} 2$ & $104(32.0)$ & $1548(32.4)$ & \\
\hline G3 & $210(64.6)$ & $2879(60.3)$ & \\
\hline Not assessed & $8(2.5)$ & $243(5.1)$ & \\
\hline Missing & $0(0.0)$ & $1(0.0)$ & \\
\hline
\end{tabular}

form of clonal anergy or immunological ignorance (16).

Our hypothesis of enhanced immune response during trastuzumab therapy for early breast cancer in patients with a history of autoimmune diseases is based on studies that demonstrated that part of the trastuzumab therapeutic effect is immune-dependent. It is possible that trastuzumab induces antibody-dependent cellular cytotoxicity (ADCC) by directing effector cells to kill HER2-expressing cancer cells (17). ADCC is directed mainly by the Fc receptor, which is present on natural killer cells that recognize the Fc portion of trastuzumab. This results in an improved response in subjects with a high percentage of TILs in their tumours and an augmented capacity to mediate ADCC (18). Other immune mechanisms involved in trastuzumab activity include: complement-dependent cytotoxicity, phagocytosis of monoclonal antibody-opsonised target cells through receptors for the $\mathrm{Fc}$ portion of $\mathrm{IgG}$, induction of production of immunomodulatory cytokines, induction of crosspresentation of tumour antigens and induction of cross-talk among immune cells $(9,19-25)$. While these mechanisms may enhance trastuzumab activity, trastuzumab treatment is not associated with increased clinical manifestations of autoimmunity (5). This may be related to the specific nature of anti-HER2 treatment and may be also the reason why the presence of autoimmune diseases is not associated with an increased benefit from trastuzumab administration. Indeed, our data suggest that autoimmune comorbidities do not influence trastuzumab effects in the adjuvant setting. This information might be helpful information for clinicians who are reluctant to provide trastuzumab to patients with autoimmune diseases.

The strengths of this study include the analysis of a homogenous population, which was observed prospectively and randomized to trastuzumab adjuvant therapy and has a 
Table III. Hazard ratios for DFS and OS of autoimmune diseases with treatment arm.

\begin{tabular}{|c|c|c|c|c|c|}
\hline & \multicolumn{3}{|c|}{ DFS } & \multicolumn{2}{|c|}{ OS } \\
\hline & Patients & Events & HR $(95 \% \mathrm{CI})$ & Events & HR $(95 \% \mathrm{CI})$ \\
\hline \multicolumn{6}{|l|}{ Overall population } \\
\hline $1+2$ years trastuzumab & 3402 & 1023 & $0.77(0.69-0.85)$ & 632 & $0.73(0.65-0.83)$ \\
\hline Observation & 1697 & 608 & - & 405 & - \\
\hline \multicolumn{6}{|l|}{ Autoimmune disease } \\
\hline $1+2$ years trastuzumab & 204 & 61 & $0.76(0.51-1.12)$ & 34 & $0.65(0.40-1.07)$ \\
\hline Observation & 121 & 43 & - & 29 & - \\
\hline \multicolumn{6}{|l|}{ No autoimmune disease } \\
\hline $1+2$ years trastuzumab & 3198 & 962 & $0.77(0.69-0.85)$ & 598 & $0.74(0.65-0.84)$ \\
\hline Observation & 1576 & 565 & & 376 & \\
\hline
\end{tabular}

long follow-up period. Moreover, the clinical data and characteristics were well annotated including the presence of autoimmune conditions. Conversely, there are limitations that should be taken into account. As this is not a pre-planned analysis, it is possible that unidentified confounders were non-randomly distributed between groups of interest. Autoantibody (such as anti-thyroglobulin) levels were not collected and information regarding autoimmunity was analyzed on the basis of baseline data only, which excluded post-treatment effects and dynamics. Another weakness of our study is that the selected cases with autoimmune disease history are composed mainly of patients with thyroid diseases especially hypothyroidism of different levels of severity which could have an influence on the end-point. Moreover, the numbers in the autoimmune group are very small; therefore, the test of interaction will be correspondingly low powered.

In conclusion, in this unplanned analysis of a prospective trial we did not confirm the hypothesis that autoimmunity could activate trastuzumab-directed responses triggering an immune response, such that the benefit from trastuzumab would be greater in such patients than those without a history of autoimmune disease.

\section{Conflicts of Interest}

The Authors have no conflicts of interest to declare regarding this study.

\section{Acknowledgements}

The Authors would like to thank all the patients who participated in the HERA trial and their families. HERA was conducted under the umbrella of the Breast international group (BIG), with sponsorship and funding provided by F Hoffmann-La Roche. The coordinating group and data centre was the Breast European Adjuvant Studies Team (BrEAST).

\section{References}

1 Slamon DJ, Clark GM, Wong SG, Levin WJ, Ullrich A and McGuire WL: Human breast cancer: correlation of relapse and survival with amplification of the HER-2/neu oncogene. Science 235: 177-182, 1987.

2 Slamon DJ, Godolphin W, Jones LA, Holt JA, Wong SG, Keith DE, Levin WJ, Stuart SG, Udove J and Ullrich A: Studies of the HER-2/neu proto-oncogene in human breast and ovarian cancer. Science 244: 707-712, 1989.

3 Slamon DJ, Leyland-Jones B, Shak S, Fuchs H, Paton V, Bajamonde A, Fleming T, Eiermann W, Wolter J, Pegram M, Baselga $\mathrm{J}$ and Norton L: Use of chemotherapy plus a monoclonal antibody against HER2 for metastatic breast cancer that overexpresses HER2. N Engl J Med 344: 783-792, 2001.

4 Piccart-Gebhart MJ, Procter M, Leyland-Jones B, Goldhirsch A, Untch M, Smith I, Gianni L, Baselga J, Bell R, Jackisch C, Cameron D, Dowsett M, Barrios CH, Steger G, Huang C-S, Andersson M, Inbar M, Lichinitser M, Láng I, Nitz U, Iwata H, Thomssen C, Lohrisch C, Suter TM, Rüschoff J, Suto T, Greatorex V, Ward C, Straehle C, McFadden E, Dolci MS, Gelber RD and Herceptin Adjuvant (HERA) Trial Study Team: Trastuzumab after adjuvant chemotherapy in HER2-positive breast cancer. N Engl J Med 353: 1659-1672, 2005.

5 Cameron D, Piccart-Gebhart MJ, Gelber RD, Procter M, Goldhirsch A, de Azambuja E, Castro G, Untch M, Smith I, Gianni L, Baselga J, Al-Sakaff N, Lauer S, McFadden E, Leyland-Jones B, Bell R, Dowsett M, Jackisch C and Herceptin Adjuvant (HERA) Trial Study Team: 11 years' follow-up of trastuzumab after adjuvant chemotherapy in HER2-positive early breast cancer: final analysis of the HERceptin Adjuvant (HERA) trial. Lancet Lond Engl 389: 1195-1205, 2017.

6 Slamon D, Eiermann W, Robert N, Pienkowski T, Martin M, Press M, Mackey J, Glaspy J, Chan A, Pawlicki M, Pinter T, Valero V, Liu M-C, Sauter G, von Minckwitz G, Visco F, Bee V, Buyse M, Bendahmane B, Tabah-Fisch I, Lindsay M-A, Riva A, Crown $\mathrm{J}$ and Breast Cancer International Research Group: Adjuvant trastuzumab in HER2-positive breast cancer. N Engl J Med 365: 1273-1283, 2011. 
7 Romond EH, Perez EA, Bryant J, Suman VJ, Geyer CE Jr., Davidson NE, Tan-Chiu E, Martino S, Paik S, Kaufman PA, Swain SM, Pisansky TM, Fehrenbacher L, Kutteh LA, Vogel VG, Visscher DW, Yothers G, Jenkins RB, Brown AM, Dakhil SR, Mamounas EP, Lingle WL, Klein PM, Ingle JN and Wolmark N: Trastuzumab plus adjuvant chemotherapy for operable HER2-positive breast cancer. N Engl J Med 353: 16731684, 2005.

8 Hoebe K, Janssen E and Beutler B: The interface between innate and adaptive immunity. Nat Immunol 5: 971-974, 2004.

9 Bellati F, Napoletano C, Ruscito I, Liberati M, Panici PB and Nuti M: Cellular adaptive immune system plays a crucial role in trastuzumab clinical efficacy. J Clin Oncol Off J Am Soc Clin Oncol 28: e369-370, 2010.

10 Bianchini $\mathrm{G}$ and Gianni L: The immune system and response to HER2-targeted treatment in breast cancer. Lancet Oncol 15: e5868, 2014.

11 Park S, Jiang Z, Mortenson ED, Deng L, Radkevich-Brown O, Yang X, Sattar H, Wang Y, Brown NK, Greene M, Liu Y, Tang J, Wang S and Fu YX: The therapeutic effect of anti-HER2/neu antibody depends on both innate and adaptive immunity. Cancer Cell 18: 160-170, 2010.

12 Loi S, Michiels S, Salgado R, Sirtaine N, Jose V, Fumagalli D, Kellokumpu-Lehtinen PL, Bono P, Kataja V, Desmedt C, Piccart MJ, Loibl S, Denkert C, Smyth MJ, Joensuu H and Sotiriou C: Tumor infiltrating lymphocytes are prognostic in triple negative breast cancer and predictive for trastuzumab benefit in early breast cancer: results from the FinHER trial. Ann Oncol Off $\mathbf{J}$ Eur Soc Med Oncol 25: 1544-1550, 2014.

13 Jacobson DL, Gange SJ, Rose NR and Graham NM: Epidemiology and estimated population burden of selected autoimmune diseases in the United States. Clin Immunol Immunopathol 84: 223-243, 1997.

14 Loi S, Sirtaine N, Piette F, Salgado R, Viale G, Van Eenoo F, Rouas G, Francis P, Crown JPA, Hitre E, de Azambuja E, Quinaux E, Di Leo A, Michiels S, Piccart MJ and Sotiriou C: Prognostic and predictive value of tumor-infiltrating lymphocytes in a phase III randomized adjuvant breast cancer trial in nodepositive breast cancer comparing the addition of docetaxel to doxorubicin with doxorubicin-based chemotherapy: BIG 02-98. J Clin Oncol Off J Am Soc Clin Oncol 31: 860-867, 2013.

15 Davidson A and Diamond B: Autoimmune diseases. N Engl J Med 345: 340-350, 2001.

16 Kamradt $\mathrm{T}$ and Mitchison NA: Tolerance and autoimmunity. N Engl J Med 344: 655-664, 2001.

17 Arnould L, Gelly M, Penault-Llorca F, Benoit L, Bonnetain F, Migeon C, Cabaret V, Fermeaux V, Bertheau P, Garnier J, Jeannin J-F and Coudert B: Trastuzumab-based treatment of HER2-positive breast cancer: an antibody-dependent cellular cytotoxicity mechanism? Br J Cancer 94: 259-267, 2006.
18 Gennari R, Menard S, Fagnoni F, Ponchio L, Scelsi M, Tagliabue E, Castiglioni F, Villani L, Magalotti C, Gibelli N, Oliviero B, Ballardini B, Da Prada G, Zambelli A and Costa A: Pilot study of the mechanism of action of preoperative trastuzumab in patients with primary operable breast tumors overexpressing HER2. Clin Cancer Res Off J Am Assoc Cancer Res 10: 5650-5655, 2004.

19 Kute TE, Savage L, Stehle JR, Kim-Shapiro JW, Blanks MJ, Wood J and Vaughn JP: Breast tumor cells isolated from in vitro resistance to trastuzumab remain sensitive to trastuzumab antitumor effects in vivo and to ADCC killing. Cancer Immunol Immunother CII 58: 1887-1896, 2009.

20 Yamauchi C, Fujii S, Kimura T, Kuwata T, Wada N, Mukai H, Matsumoto N, Fukayama $\mathrm{M}$ and Ochiai A: E-cadherin expression on human carcinoma cell affects trastuzumabmediated antibody-dependent cellular cytotoxicity through killer cell lectin-like receptor G1 on natural killer cells. Int J Cancer 128: 2125-2137, 2011.

21 Nimmerjahn F and Ravetch JV: Fcgamma receptors as regulators of immune responses. Nat Rev Immunol 8: 34-47, 2008.

22 Tamura K, Shimizu C, Hojo T, Akashi-Tanaka S, Kinoshita T, Yonemori K, Kouno T, Katsumata N, Ando M, Aogi K, Koizumi F, Nishio K and Fujiwara Y: Fc $\gamma R 2 A$ and 3A polymorphisms predict clinical outcome of trastuzumab in both neoadjuvant and metastatic settings in patients with HER2-positive breast cancer. Ann Oncol Off J Eur Soc Med Oncol 22: 1302-1307, 2011.

23 Cooley S, Burns LJ, Repka T and Miller JS: Natural killer cell cytotoxicity of breast cancer targets is enhanced by two distinct mechanisms of antibody-dependent cellular cytotoxicity against LFA-3 and HER2/neu. Exp Hematol 27: 1533-1541, 1999.

24 Capietto AH, Martinet L and Fournié JJ: Stimulated $\gamma \delta$ T cells increase the in vivo efficacy of trastuzumab in HER-2+ breast cancer. J Immunol Baltim Md 1950 187: 1031-1038, 2011.

$25 \mathrm{Lu} \mathrm{H}$, Yang Y, Gad E, Inatsuka C, Wenner CA, Disis ML and Standish LJ: TLR2 agonist PSK activates human NK cells and enhances the antitumor effect of HER2-targeted monoclonal antibody therapy. Clin Cancer Res Off J Am Assoc Cancer Res 17: 6742-6753, 2011.
Received December 13, 2018

Revised January 8, 2019

Accepted January 10, 2019 